\title{
Host Immune Response Triggered by Graphene Quantum-Dot-Mediated Photodynamic Therapy for Oral Squamous Cell Carcinoma
}

This article was published in the following Dove Press journal: International Journal of Nanomedicine

\author{
Xiliu Zhang' \\ Hongyu $\mathrm{Li}^{1}$,* \\ Chen $\mathrm{Yi}^{\mathrm{l}, *}$ \\ Guosheng Chen ${ }^{2}$ \\ Ye $\mathrm{Li}^{\prime}$ \\ Ying Zhou' \\ Guanhui Chen' \\ Yiming Li' \\ Yi Hel \\ Dongsheng Yu' \\ 'Guanghua School of Stomatology, \\ Hospital of Stomatology, Sun Yat-Sen \\ University, Guangdong Provincial Key \\ Laboratory of Stomatology, Guangzhou \\ 5 I0055, People's Republic of China; \\ ${ }^{2}$ MOE Key Laboratory of Bioinorganic \\ and Synthetic Chemistry, School of \\ Chemistry, Sun Yat-Sen University, \\ Guangzhou 510275, People's Republic of \\ China
}

*These authors contributed equally to this work

\begin{abstract}
Introduction: With the innovation of photosensitizers, photodynamic therapy is now widely used in antitumor detection and treatment. Graphene quantum dots (GQDs) are proposed as a promising alternative photosensitizer due to their high biocompatibility, specific photoactivity, and strong tumor concentration. However, the changes in host immunity triggered by GQDs have only rarely been reported.

Methods: In this work, GQDs as photosensitizers were conjugated to polyethylene glycol (PEG) to enhance solubility and blood circulation. The phototoxicity of the resulting GQD-PEG nanomaterials was then detected in vitro and in vivo. The antitumor immunity triggered by GQDPEG under irradiation was further evaluated in an oral squamous cell carcinoma animal model. Results: The obtained GQD-PEG nanomaterials exhibited low cytotoxicity, good solution stability, and excellent endocytosis. Both in vitro and in vivo, all demonstrated strong ablation for oral squamous cell carcinoma under irradiation. Meanwhile, host-immunityrelated $\mathrm{CD}^{+} \mathrm{T}$ cells (cytotoxic $\mathrm{T}$ lymphocytes) and proinflammatory cytokines, including IFN- $\gamma$ and TNF- $\alpha$, were significantly increased after photo-activated antitumor activity.

Conclusion: These results highlight the dominant role of GQD-PEG in photodynamic therapy and could have significant implications for further combination therapy as a promising antitumor immune response strategy triggered by nanomaterials.
\end{abstract}

Keywords: graphene quantum dots, photodynamic therapy, host immunity, oral squamous cell carcinoma

\section{Introduction}

Cancer immunotherapy is emerging as a promising treatment, with gradually increasing mortality of cancers and decreasing morbidity of young patients. ${ }^{1,2}$ It relies on intervening in the body's inherent immunological system and augmenting antitumor responses. Because of its specific noninvasiveness and fewer side-effects, immunotherapy has become a powerful clinical strategy for treating various tumor types. However, it is still characterized by high cost, drug resistance, and limited efficacy in particular patients or tumor types. ${ }^{3,4}$ Therefore, there is great interest in the development of a combination treatment strategy to enhance immune response. ${ }^{5}$

Photodynamic therapy (PDT) is a novel option for tumor ablation compared with conventional antitumor approaches such as chemotherapy and radiotherapy. As a well-established and photoactivated method, direct cancer ablation is based on the abundant toxicity of reactive oxygen species (ROS). Furthermore, a "domino effect" immune response is triggered by tumor-associated antigens released by
Correspondence: Dongsheng Yu Hospital of Stomatology, Sun Yat-Sen University, Guangdong Provincial Key Laboratory of Stomatology, Guangzhou 510055, People's Republic of China Email yudsh@mail.sysu.edu.cn 
apoptosis or necrosis cells. Multiple immune mechanisms are activated in the tumor microenvironment, inducing acute inflammation and antigen-presenting cells (APC) infiltration, further leading to the activation of $\mathrm{T}$ cells and augmenting host antitumor immunity. ${ }^{6,7}$ Several studies have concentrated on photodynamic immunotherapy combined with an immune checkpoint blockade for tumor therapy. Wang et $\mathrm{al}^{8}$ demonstrated that the combination of a PD-L1 blockade and PDT therapy exhibited significantly enhanced efficacy for the inhibition of primary and distant tumor growth, compared with either treatment alone. $\mathrm{Yu}$ et $\mathrm{al}^{9}$ synthesized GO (HPPH)-PEG-HK particles as photosensitizers and observed not only primary tumor ablation but also distant lung metastasis suppression by the stimulation of dendritic cells (DCs) and the infiltration of $\mathrm{CD}^{+} \mathrm{T}$ lymphocytes. Recent studies found that nanoparticles could be designed for a tumor microenvironment and increasing tissue penetration. Compared with $100-\mathrm{nm}$ nanoparticles, 10-nm quantum dots facilitated lower immunogenicity, which could be used to optimize therapeutic-loaded nanoparticles. ${ }^{10,11}$

Graphene quantum dots (GQDs), zero-dimension graphene-derived materials, have a wide range of applications in the field of biomedicine due to their excellent physical and chemical properties. Currently, GQDs are attracting more interest in phototherapy, bioimaging, and drug delivery, due to their unique and tunable photoluminescence, derived from quantum confinement and edge effects. ${ }^{12}$ Upon specific light-activation, GQDs have been proven to produce singlet oxygen and other ROS, which is key to the phototoxicity for PDT. ${ }^{13,14}$ In addition, self-targeting in tumor tissue and the tunable emission wavelength of GQDs are of benefit for imaging and theranostics, ${ }^{15,16}$ and the high water solubility and functional groups also render GQDs to be suitable drug nanocarriers. ${ }^{17}$ It is reasonable to suppose that GQD-mediated photo-immunotherapy may emerge as a new antitumor strategy. Nevertheless, limited efforts have been devoted to addressing this topic.

Hence, in the present work, a hybrid photosensitizer based on pristine GQDs connected with polyethylene glycol (PEG) was proposed for applying PDT and evaluating the systematic immune change post-PDT. The obtained GQD-PEG exhibited dramatic ROS-generation efficacy and excellent biocompatibility under 560-nm laser irradiation. Meanwhile, both in vitro and in vivo study verified that the GQD-PEG showed strong ablation for oral squamous cell carcinoma under irradiation, compared with the control groups. We further demonstrated that antitumor immune-related cytotoxic $\mathrm{T}$ lymphocytes (CTL) and proinflammatory cytokines, including IFN- $\gamma$ and TNF- $\alpha$, were significantly increased after phototherapy (Figure 1). These results indicated that GQD-mediated photoimmunotherapy may become a good candidate for antitumor therapy.

\section{Experimental Protocol Materials}

GQDs (100 mg, red fluorescence) were purchased from XFNANO Materials Tech Co., Ltd (Nanjing, China). $\mathrm{NH}_{2}-$ PEG-NH $\mathrm{N}_{2}(\mathrm{Mw}=4 \mathrm{kDa})$ was obtained from SINOPEG (Xiamen, China).

$\mathrm{N}$-(3-Dimethylaminopropyl)-N'-ethylcarbodiimide hydrochloride (EDC; 98\%) and N-Hydroxysuccinimide (NHS; 98\%) were purchased from aladdin (Shanghai, China). Singlet oxygen sensor green (SOSG) was obtained from Molecular Probes (Eugene, OR, USA). Fluorochromelabeled CD3, CD4, and CD8 monoclonal antibodies were purchased from eBioscience (San Diego, CA, USA). Enzymelinked immunosorbent assays (ELISA) of TNF- $\alpha$ and IFN- $\gamma$ antibodies were also obtained from eBioscience.

\section{Cell Lines and Animal Model}

The human oral squamous cell carcinoma cell lines SCC 25 and SCC 9 and the normal cell line, human oral keratinocyte (HOK), were purchased from the ATCC and used for in vitro experiments. The murine SCC cell line SCC VII were used for in vivo experiments, which was provided by Professor Yixiang Wang, School of Stomatology, Peking University. Wild-type C3H mice (6to 8-week-old females; weight, 18-20 g) were purchased from Vital River Laboratory Animal Technology Co., Ltd. (Beijing, China). The animal model for OSCC was established by subcutaneous injection of SCC VII cells into the right armpit of each mouse at $3.0 \times 10^{6}$ cells/mouse. The animal use based on SCC VII cells has been reviewed and approved by the Institutional Animal Care and Use Committee (IACUC), Sun Yat-Sen University (Approval Number: 2,019,000,249).

\section{Synthesis of GQD-PEG}

In the typical process, sonicate solution was initially used for 30 minutes at $700 \mathrm{~W}$ to achieve the necessary GQD dispersion. A 100-mg quantity of EDC and a 21.5-mg quantity of NHS were added to $2 \mathrm{mg} / \mathrm{mL}$ GQD solution for $2 \mathrm{~h}$ for activation of the carboxylic groups presenting on the surfaces 


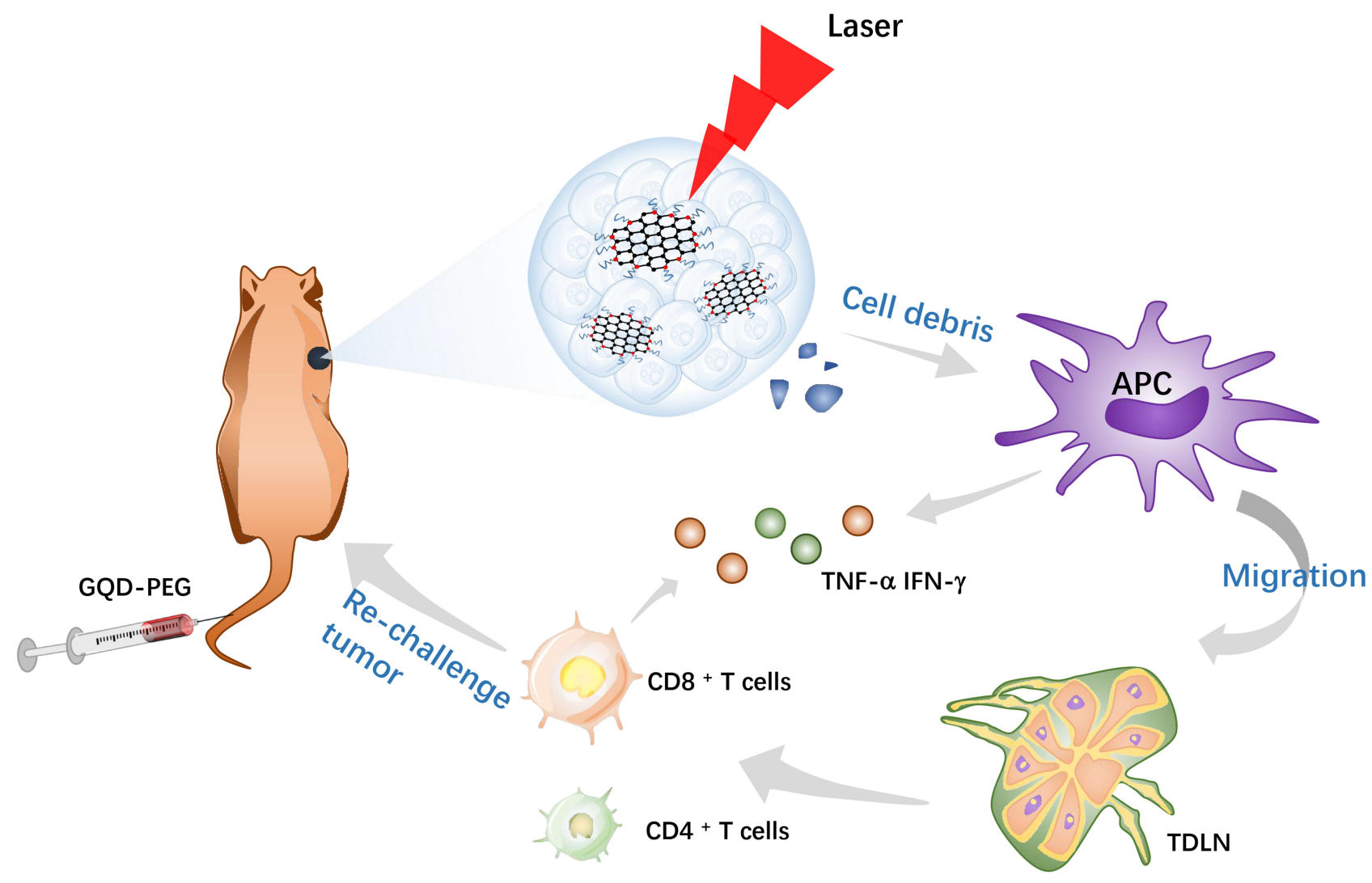

Figure I Schematic illustration of GQD-PEG-mediated photo-triggered immune responses for tumor therapy.

of GQDs. Then, a 250-mg quantity of $\mathrm{NH}_{2}-\mathrm{PEG}-\mathrm{NH}_{2}$ was added to the solution and stirred overnight. For removal of the unattached PEG, the resulting mixture underwent dialysis for $72 \mathrm{~h}$ in $7 \mathrm{kDa}$ dialysis membrane against water. Throughout, exposure to direct and strong light was avoided. The morphologies of GQD-PEG were visualized by transmission electron microscopy (TEM). Elemental composition and surface functional groups were analyzed by energydispersive spectroscopy (EDS) and Fourier transform infrared spectroscopy (FTIR). UV-vis absorption and fluorescence intensity were measured by UV-vis spectrophotometry.

\section{Cellular Uptake}

Cells were grown in DMEM/F12 (Gibco, Thermo Fisher Scientific, Inc., Waltham, MA, USA) supplemented with $10 \%$ fetal bovine serum (FBS; Gibco, Thermo Fisher Scientific, Inc.) at $37^{\circ} \mathrm{C}$ in a humidified atmosphere of $5 \%$ $\mathrm{CO}_{2}$. For cell viability, cells were seeded in 96-well plates and cultured for $24 \mathrm{~h}$, and then replaced with fresh medium containing series concentrations of GQD-PEG for another 12 h. A portion of viable cells was estimated with the Cell Counting Kit-8 assay (CCK-8). For observation of the cellular uptake of GQD-PEG, SCC 9 and human oral keratinocytes (HOK) were seeded in confocal Petri dishes $(15 \mathrm{~mm})$ at a density of $10^{4}$ cells $/ \mathrm{mL}$ and cultured for 24 h. Cells were then incubated with GQD-PEG or pristine GQD at a concentration of $50 \mu \mathrm{g} / \mathrm{mL}$ for $6 \mathrm{~h}$ and washed 3 times with phosphate-buffered saline (PBS; Hyclone, Logan, UT, USA). For the tracking of intracellular distribution, cells were stained with 4',6-diamidino-2-phenylindole (DAPI) and observed by confocal laser scanning microscopy (CLSM; Zeiss, Jena, Germany). Fluorescence signals of nanomaterials were measured at a $560-\mathrm{nm}$ excitation wavelength.

\section{Singlet Oxygen Generation}

The SOSG assay was utilized to detect the singlet production of ${ }^{1} \mathrm{O}_{2}$ by CLSM. For dose-dependent singlet oxygen generation, cells in 96-well plates were incubated with GQD-PEG for $50 \mu \mathrm{g} / \mathrm{mL}$ and then irradiated in $560 \mathrm{~nm}$ for $0,1,2,3,5,10$, and 15 minutes. The fluorescence signals of SOSG were detected by fluorimetric photometry at an excitation wavelength of $490 \mathrm{~nm}$.

\section{In vitro Photodynamic Therapy}

For the assessment of antitumor activity of GQD-PEG, cells were seeded in 24-well plates at a density of 
$3.0 \times 10^{4}$ cells $/ \mathrm{mL}$ and cultured with DMEM/F12. Twentyfour $h$ later, cells were treated with GQD-PEG in a series of concentrations $(25,50$, and $100 \mu \mathrm{g} / \mathrm{mL})$ for $12 \mathrm{~h}$ and subjected to laser irradiation for 10 minutes. Following another 24-hour incubation, cell proliferation ability and cellular apoptosis were detected by the CCK-8 assay and flow cytometry, respectively. Cellular apoptosis was also visualized by means of a live/dead cell fluorescence staining assay (double-staining with calcein-AM and PI).

\section{In vivo Imaging}

The animal model for OSCC was established by subcutaneous injection of SCC VII cells into the right armpits of mice at $3.0 \times 10^{6}$ cells/mouse. SCC VII tumor-bearing $\mathrm{C} 3 \mathrm{H}$ mice ( $\mathrm{n}=3$ per group) were intravenously injected with GQD-PEG at $200 \mu \mathrm{g}$ per mouse. Tumor-bearing mice treated with PBS were utilized as controls. Four and $24 \mathrm{~h}$ post-injection, the mice were anesthetized by isoflurane, and their bodies were shaved. Fluorescence signals were detected by Xenogen IVIS Spectrum (Caliper Life Sciences, Hopkinton, MA, USA). The tumor uptake of GQD-PEG was measured by qualifying the fluorescence intensity of ROI with IVIS Spectrum software (Caliper Life Sciences, Hopkinton, MA, USA).

\section{In vivo Phototoxicity and Immune Response}

SCC VII tumor-bearing $\mathrm{C} 3 \mathrm{H}$ mice were divided into 3 groups ( $\mathrm{n}=5$ per group), those treated with PBS, those treated with PBS and laser only, and those treated with GQD-PEG and laser. Mice were intravenously injected with $200 \mu \mathrm{g}$ GQD-PEG and laser-irradiated $\left(1 \mathrm{w} / \mathrm{cm}^{2}, 8\right.$ minutes) $4 \mathrm{~h}$ post-injection 3 times every 2 days. Tumor sizes were recorded every two days. Tumor volume was calculated in accordance with the equation: $\mathrm{V}=\mathrm{W}^{2} \mathrm{~L} / 2$ (W, shortest diameter; L, longest diameter).

When tumors reached about $150 \mathrm{~mm}^{3}$, the mice of all groups were sacrificed. Tumors were fixed with $4 \%$ paraformaldehyde for immunofluorescence detection. Tumordraining lymph nodes from all 3 groups were isolated and digested to form a single-cell suspension. Cells were then stained with antibodies against CD3 (APC-Cyanine7), CD4 (FITC), and CD8 (PE), and then analyzed by flow cytometry. IFN- $\gamma$ and TNF- $\alpha$ in the serum samples were collected and analyzed by ELISA kits. The body weights of $\mathrm{C} 3 \mathrm{H}$ mice were measured every 2 days. At the end of the experimental process, the major organs of the mice in each group were harvested and sectioned into slices for histopathological evaluation, with $\mathrm{H} \& \mathrm{E}$ staining.

\section{Statistical Analysis}

All data are presented as means \pm standard errors. Statistically significant differences were analyzed by oneway analysis of variance or Tukey's multiple-comparisons test when appropriate. $P<0.05$ was considered a statistically significant difference. GraphPad Software 7.0 (GraphPad Software Inc., La Jolla, CA, USA), Origin pro 9.1 (OriginLab Corporation, Northampton, MA, USA), and GM 3.9 (Gatan DigitalMicrograph, Pleasanton, CA, USA) were used for statistical analyses. Every experiment was repeated at least 3 times.

\section{Results and Discussion Preparation and Characterization of GQD-PEG}

The preparation process for GQD-PEG is illustrated in Figure 2A. Functionalization with PEG was shown to significantly improve the water solubility and enhance the biocompatibility of GQDs. ${ }^{18,19}$ Herein, the appropriate molecular weight of $\mathrm{NH}_{2}$-PEG-NH $\mathrm{NH}_{2}\left(\mathrm{M}_{\mathrm{w}}=4000 \mathrm{Da}\right)$ was chemically bonded with the carboxyl edges of GQDs. The obtained monodispersed GQD-PEG was observed under TEM imaging (Figure 2B). Particle size distribution measured by dynamic light-scattering indicated that the diameters of GQD-PEG were from $3 \sim 4 \mathrm{~nm}$, in agreement with the results of TEM imaging (Figure 2C). The results obtained from the EDS spectrum were consistent with the expected elemental composition for GQD-PEG (Supporting Information, Figure S1), which confirmed the formation of $\mathrm{N}, \mathrm{C}$, and $\mathrm{O}$ as expected. ${ }^{19}$ As shown in Figure 2D, the successful integration of PEG moieties with GQD was confirmed by FTIR. The absorption band at $1652 \mathrm{~cm}^{-1}$, $1780 \mathrm{~cm}^{-1}$, and $3426 \mathrm{~cm}^{-1}$ was assigned to the aromatic $\mathrm{C}=\mathrm{C}, \mathrm{C}=\mathrm{O}, \mathrm{O}-\mathrm{H}$ stretching peak of $\mathrm{GQD},{ }^{20}$ and the new characteristic band that emerged at $2887 \mathrm{~cm}^{-1}$ and $1113 \mathrm{~cm}^{-1}$ (C-H and $\mathrm{C}-\mathrm{O}-\mathrm{C}$ groups) was assigned to the integrated PEG moieties, respectively. As shown in Figure S2, GQDPEG exhibited remarkable stability compared with pristine GQDs in all biological solutions (saline solution, PBS, serum, and DMEM), owing to the stabilizing effect of PEG. GQD-PEG inherited the UV absorption characteristics similar to those of GQDs (Figure S3), indicating the structural maintenance of GQDs after modification. In addition, the photoluminescence intensity of the GQDs (Figure 2E) in 
A

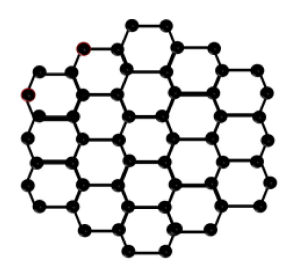

EDC/NHS

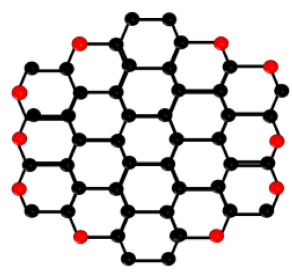

PEGylation

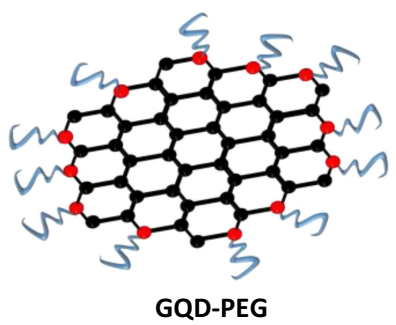

हित्: GQD

Carboxyl

W PEG

B

C

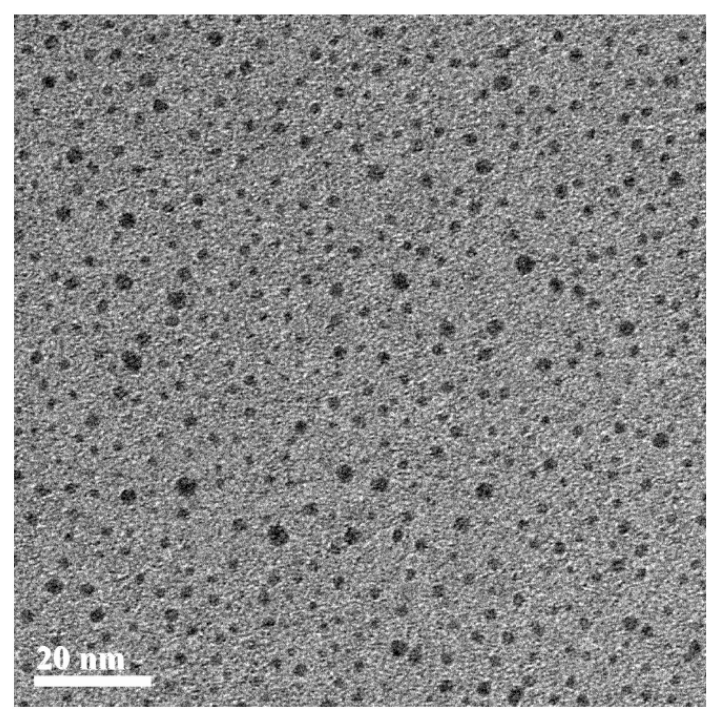

D
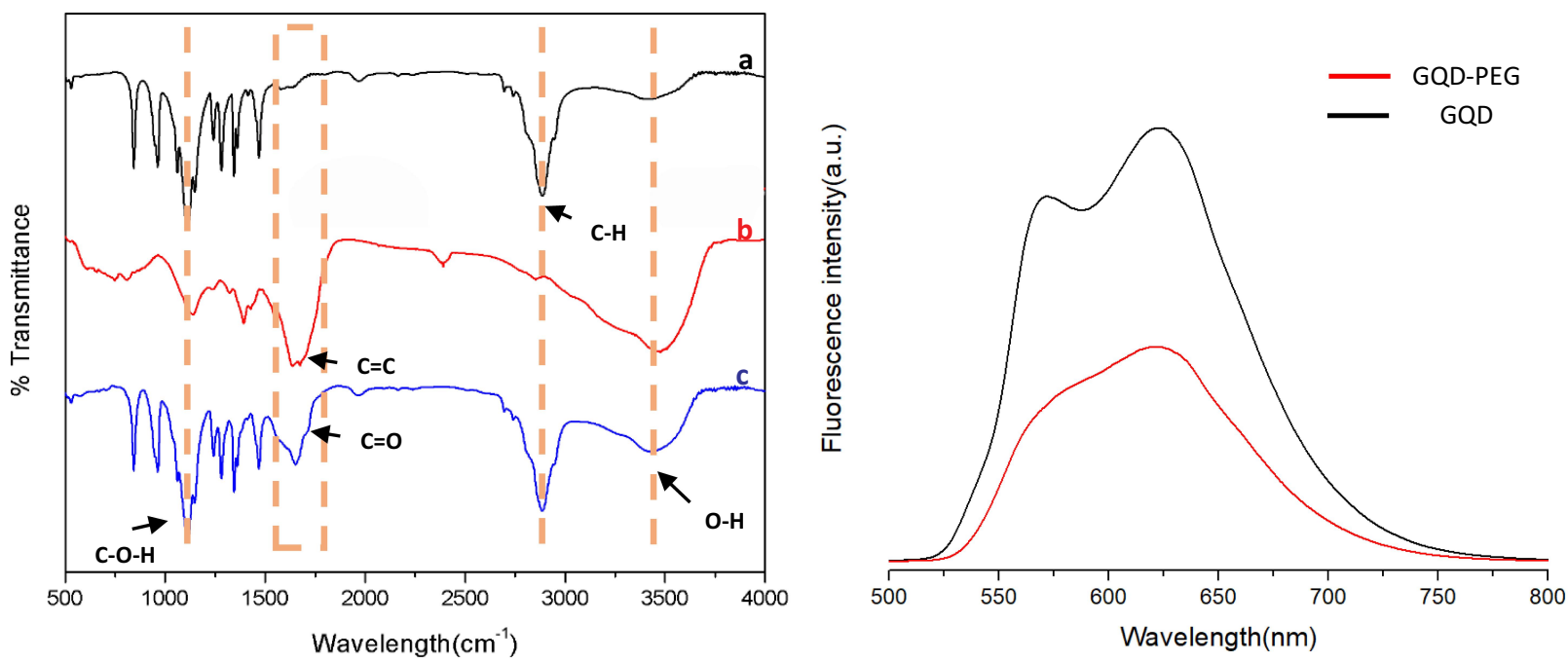

Figure 2 Characterization of GQD-PEG. (A) The preparation process of GQD-PEG. (B) TEM image of GQD-PEG. (C) Diameter distribution of GQD-PEG. (D) FTIR spectrum of (a) PEG, (b) GQD, and (c) GQD-PEG. (E) PL spectra of GQD-PEG at 560-nm excitation. 
solution was decreased by PEG functionalization, which might result from the charge-transfer to the edges of GQDs after PEG functionalization. ${ }^{18}$ Together, these results verified that the monodispersed GQD-PEG was successfully constructed.

\section{In vitro Photodynamic Efficacy of GQD-PEG}

GQDs are not only an alternative for photodynamic therapy, but also a promising carrier for immune drugs. ${ }^{21,22}$ For evaluation of the therapeutic efficacy of GQD-PEG, the cytotoxicity of the resulting compound was evaluated in human SCC 9 and SCC 25 cells (Figures S4A, S4B). The cytotoxicity of GQD-PEG is dose-dependent, according to a CCK-8 assay reported previously. ${ }^{23}$ After 12-hour incubation in the dark, the GQD-PEG group exhibited high cell viability for both SCC 9 and SCC 25 cells, even at high concentrations $(100 \mu \mathrm{g} / \mathrm{mL})$. Compared with the control group, cell viability of GQD-PEG groups was maintained at above $80 \%$ when the concentration was below $100 \mu \mathrm{g} / \mathrm{mL}$. Therefore, we chose GQD-PEG concentrations below 100 $\mu \mathrm{g} / \mathrm{mL}$ for the following in vitro study. Significant PDT efficacy of GQD-PEG was displayed upon laser irradiation (Figure 3A and B). After incubation with $100 \mu \mathrm{g} / \mathrm{mL}$ and light irradiation for 10 minutes, SCC 9 and SCC 25 cells showed variability decreases of $70 \%$ and $30 \%$, respectively, in contrast to control groups. Meanwhile, a live/dead cell fluorescence staining assay further indicated that the photodynamic therapy group based on GQD-PEG was more effective in killing SCC 9 cells than were control groups with different concentrations, which was consistent with the CCK-8 assay (Figure 3C). As shown in Figure S5, analysis also demonstrated that early apoptosis and late apoptosis were increased to $5.31 \%$ and $5.12 \%$, respectively, compared with the PBS group $(0.10 \%$ and $0.05 \%)$ after treatment with $25 \mu \mathrm{g} / \mathrm{mL}$ concentration of GQD-PEG. These results all indicated the high phototoxicity of GQD-PEG in vitro.

Since specific targeting for tumor cells was essential for PDT efficacy, cellular internalization of GQD-PEG was investigated in SCC 9 and HOK. Figure 3D displays a panel of confocal fluorescent images of GQD-PEG and pristine GQD after $6 \mathrm{~h}$ of incubation. For tumor cell, both pristine GQD and GQD-PEG all exhibited similar strong accumulation in tumor cell nuclei and cytoplasm. Nonetheless, pristine GQD exhibited stronger accumulation in normal cell nuclei compared with GQD-PEG nanomaterials. This specific and differential accumulation may help further GQD-PEG applications in the field of phototherapy through the protection of normal tissue. As expected, SOSG detection further illustrated the strong phototoxicity of GQD-PEG under laser irradiation. GQDPEG exhibited stronger ${ }^{1} \mathrm{O}_{2}$ production ability with increased irradiation (Figure 3E). Further, GQD-PEG exhibited significant potential as a light therapeutic agent for in vivo antitumor applications. In addition, GQD-PEG continued to exhibit high efficiency in PDT performance even with the interference of energy resonance transfer upon PEG functionalization.

\section{In vivo Optical Imaging and Biodistribution Study}

The carbon-dot-mediated nanoplatform has been shown to have the ability to increase the cellular uptake of nanoparticles and reduce unwanted side-effects. ${ }^{24-26}$ Here, we report the first study of the biodistribution of GQD-PEG in SCC VII-bearing $\mathrm{C} 3 \mathrm{H}$ mice, a classic animal model for OSCC. ${ }^{27-29}$ From the in vivo imaging (Figure 4A), it can be seen that the main fluorescence signals of GQD-PEG groups are located primarily in the tumor tissue compared with those of the control groups. Four $h$ after injection, the intensity of fluorescence signals in the tumor was the strongest, and a strong fluorescence signal was still observed up to $24 \mathrm{~h}$. These findings may be because of the enhanced uptake ability and solution dispersion of GQD-PEG. The timely dissection of the main organs also demonstrated the existence of strong fluorescence in the tumor, whereas some fluorescence was observed in the liver and kidney (Figure 4B and C). These fluorescence signals detected in tumor and other tissues were probably due to the significant accumulation in the tumor and the slow metabolism of GQD-PEG. Specifically, GQD-PEG was able to accumulate efficiently in tumor areas and exhibit excellent pharmacokinetics, which was beneficial for PDT and drug delivery in vivo. ${ }^{30}$

\section{In vivo Photoimmune Synergistic Antitumor Efficacy of GQD-PEG}

Various studies have reported that non-invasive PDT was capable of inducing an effective response in a portion of OSCC without the risk of any serious side-effects. ${ }^{31-33}$ These advantages might help establish PDT as the routine treatment for some OSCC, along with radiotherapy and chemotherapy. However, the widespread use of PDT requires more efficient phototoxicity and the potential of combination therapy. Encouraged by the antitumor effects 
A

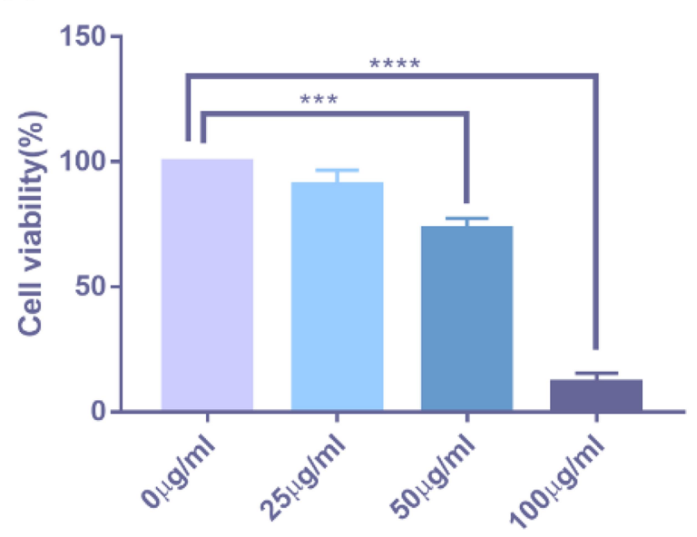

Concentration $(\mu \mathrm{g} / \mathrm{ml})$

C
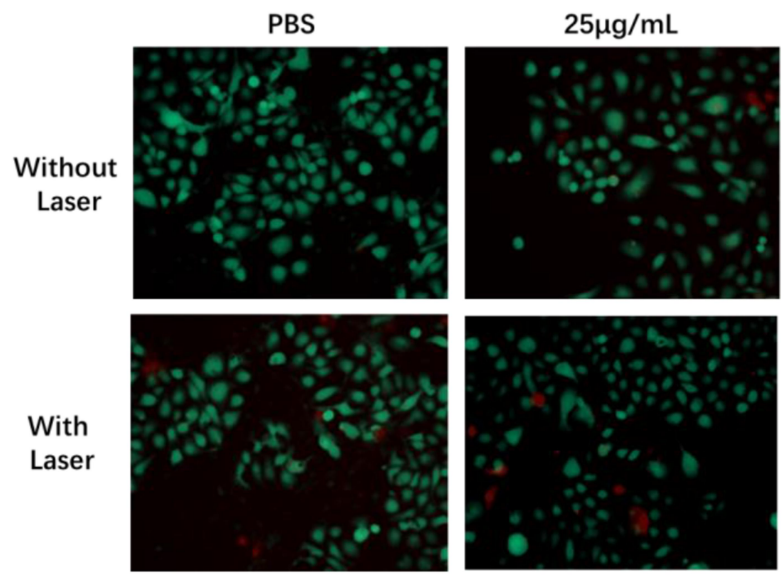

D
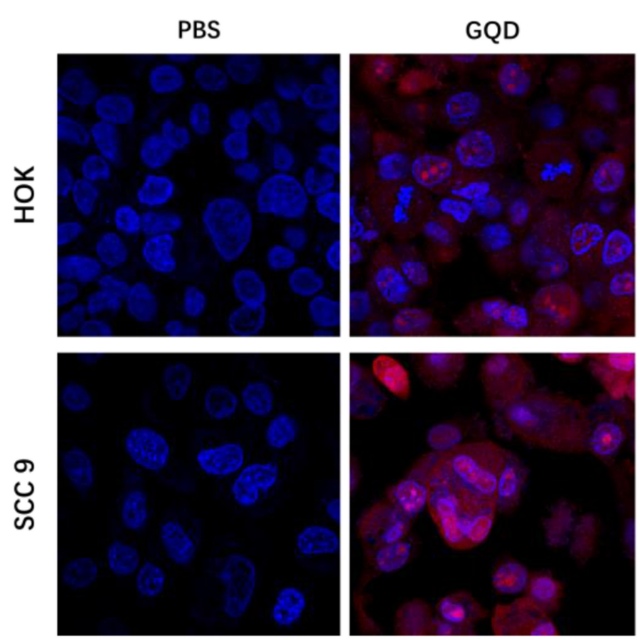

B

\section{$\operatorname{scc} 25$}

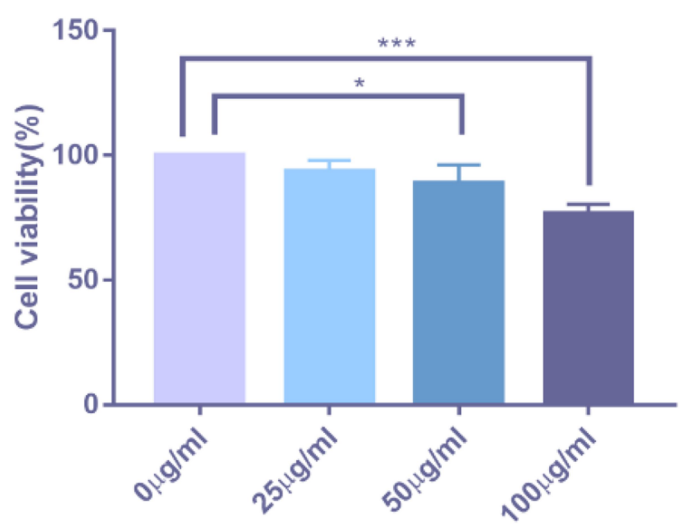

Concentration $(\mu \mathrm{g} / \mathrm{ml})$
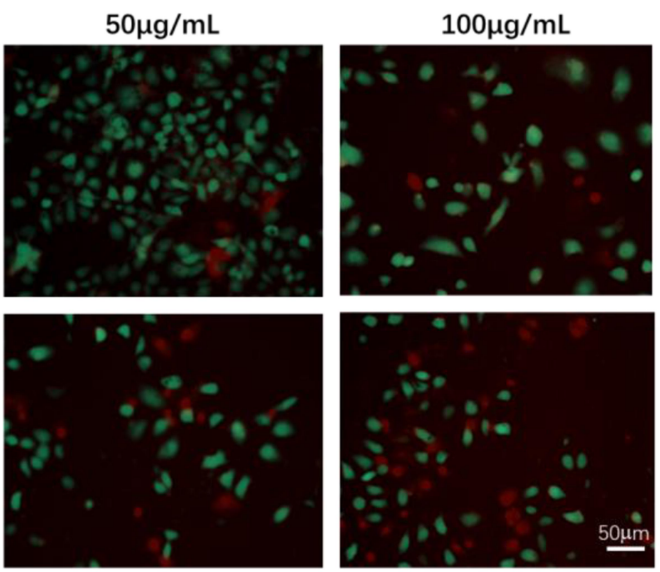

E
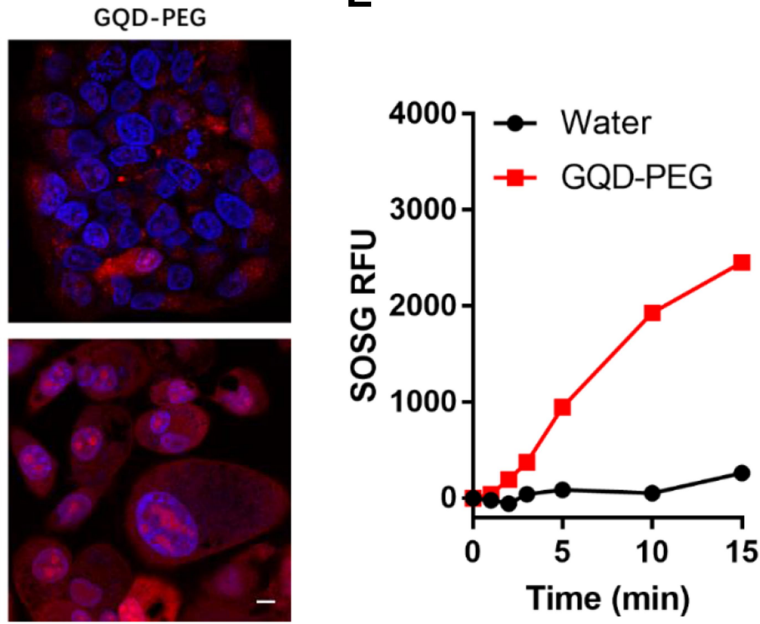

Figure 3 In vitro cytotoxicity of GQD-PEG. Cell viability of SCC 9 cells (A) and SCC 25 cells (B) incubated with GQD-PEG at different concentrations under irradiation. (C) Detection of cell viability using fluorescence probe (dead cells: red fluorescence of PI; live cells: green fluorescence of calcein). (D) Representative CLSM micrographs treated with pristine GQD and GQD-PEG for $6 \mathrm{~h}$. Scale bar: $10 \mu \mathrm{m}$. (E) Singlet oxygen generation of GQD-PEG upon irradiation $\left(560 \mathrm{~nm}, \mathrm{I}\right.$ W/cm $\left.{ }^{2}\right)$. $* P<0.05 ; * * * P<0.00$ I; $* * * * P<0.0001$. 


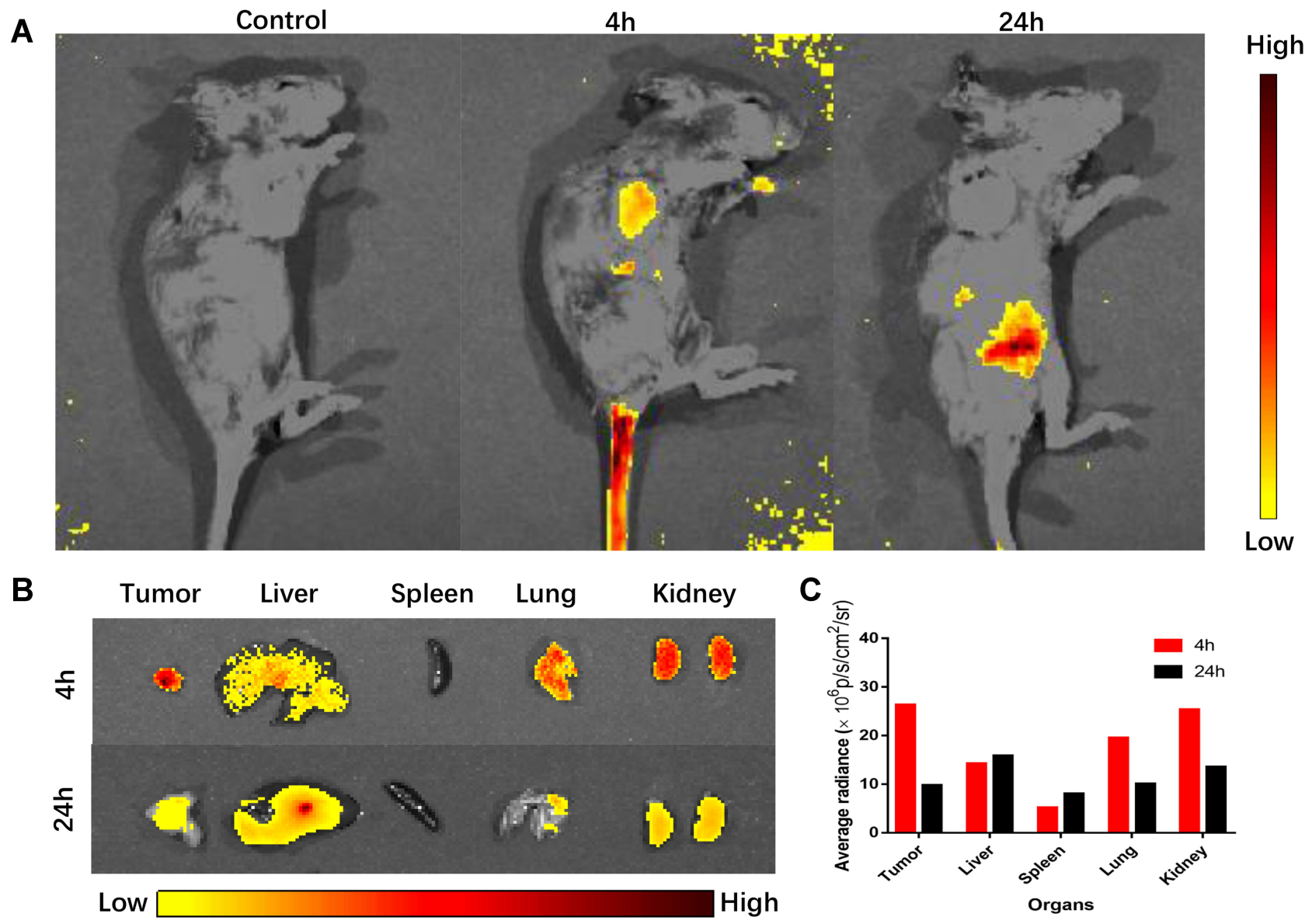

Figure 4 In vivo fluorescence imaging of GQD-PEG. (A) In vivo biodistribution of C3H mice after intravenous injection with GQD-PEG for $24 \mathrm{~h}, 4 \mathrm{~h}$ and $24 \mathrm{~h}$ post injection. (B) Ex vivo fluorescence imaging of major organs and (C) mean fluorescence intensity of the excised organs.

of PDT based on GQD-PEG in vitro and its remarkable tumor-targeting properties in vivo, we further investigated the antitumor efficiency of GQD-PEG with irradiation in SCC VII tumor-bearing C3H mice. Three groups, including PBS, PBS + laser, and GQD-PEG + laser, were evaluated once every other day on three consecutive occasions (Figure 5A). The tumor sizes and body weights of $\mathrm{C} 3 \mathrm{H}$ mice were measured continuously during this process. Figure 5B displays the curves of tumor growth. The GQDPEG group showed significantly inhibited tumor growth compared with the control and laser groups. The "GQDPEG plus irradiation" group elicited tumor size reduction exceeding $70 \%$, compared with those of the "laser only" and "PBS plus laser" groups. The direct antitumor efficacy might be attributable to the efficient tumor accumulations mediated by the enhanced permeability and retention (EPR) effect and the high ${ }^{1} \mathrm{O}_{2}$ toxicity induced from photoactivity. The average tumor weights were measured as $0.75 \mathrm{~g}, 0.45 \mathrm{~g}$, and $0.11 \mathrm{~g}$ for groups treated with PBS, laser, and GQD-PEG plus irradiation, respectively
(Figure 5C). The tumor images showed the excellent therapeutic efficacy of GQD-PEG with irradiation (Figure 5D). Figure 5E shows the changes of body weight of all treated mice. Because of the appropriate doses and intensities of treatments utilized, the weights of the mice showed slight increases with the different treatments.

Immune therapy as an emerging treatment was seen as an excellent combination therapy for PDT antitumor treatment because it is non-invasive and repeatable. Various nanoparticles have been demonstrated in specific photo-triggered tumor immunotherapy, as has their "domino effect" in the tumor microenvironment. ${ }^{34-36}$ Without irradiation, GQDs have been proven to suppress proinflammatory $\mathrm{T}$ cell responses by potentiating the tolerogenic DC functions via the induction of autophagy. ${ }^{37}$ A recent study also demonstrated that GQDs could alleviate immune-mediated central nervous system damage by modulating immune responses. ${ }^{38}$ However, there has been no research focused on the GQD-PEG-mediated systematic immune response with irradiation. Here, the immunostimulatory activity of GQD-PEG was detected by 
A

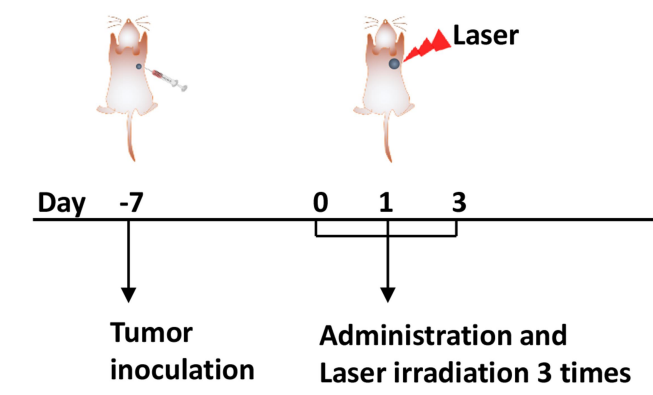

C

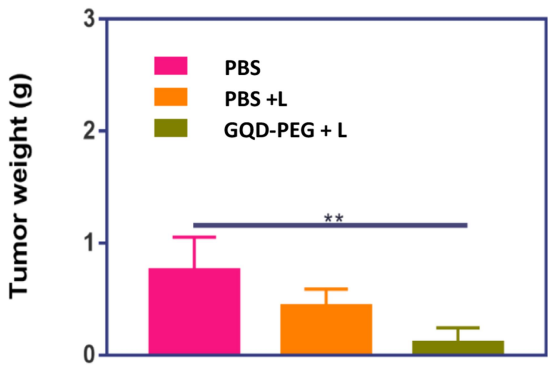

$\mathbf{F}$

D

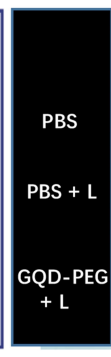

DAPI

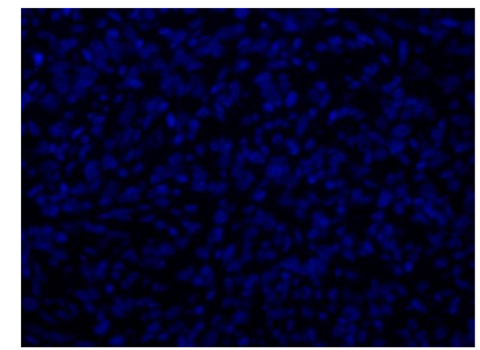

PBS

PBS + L
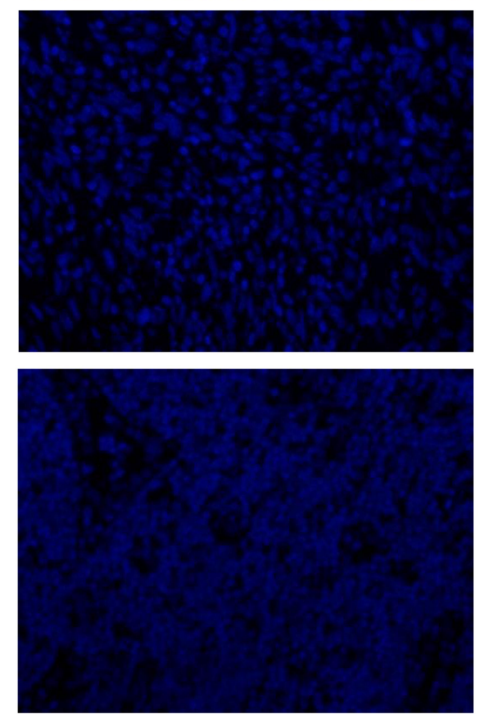

B

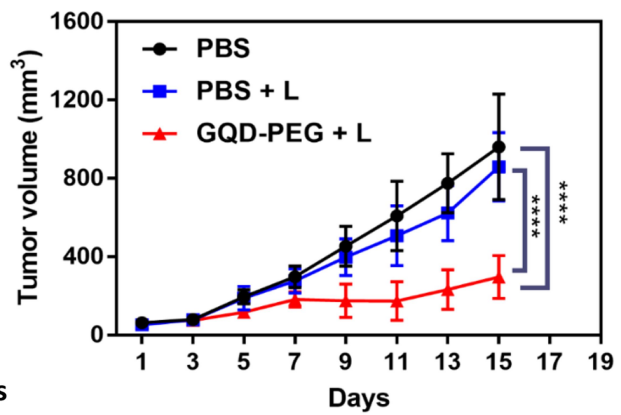

E

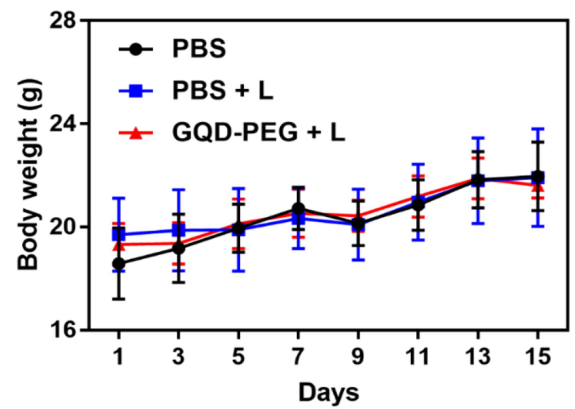

Merge
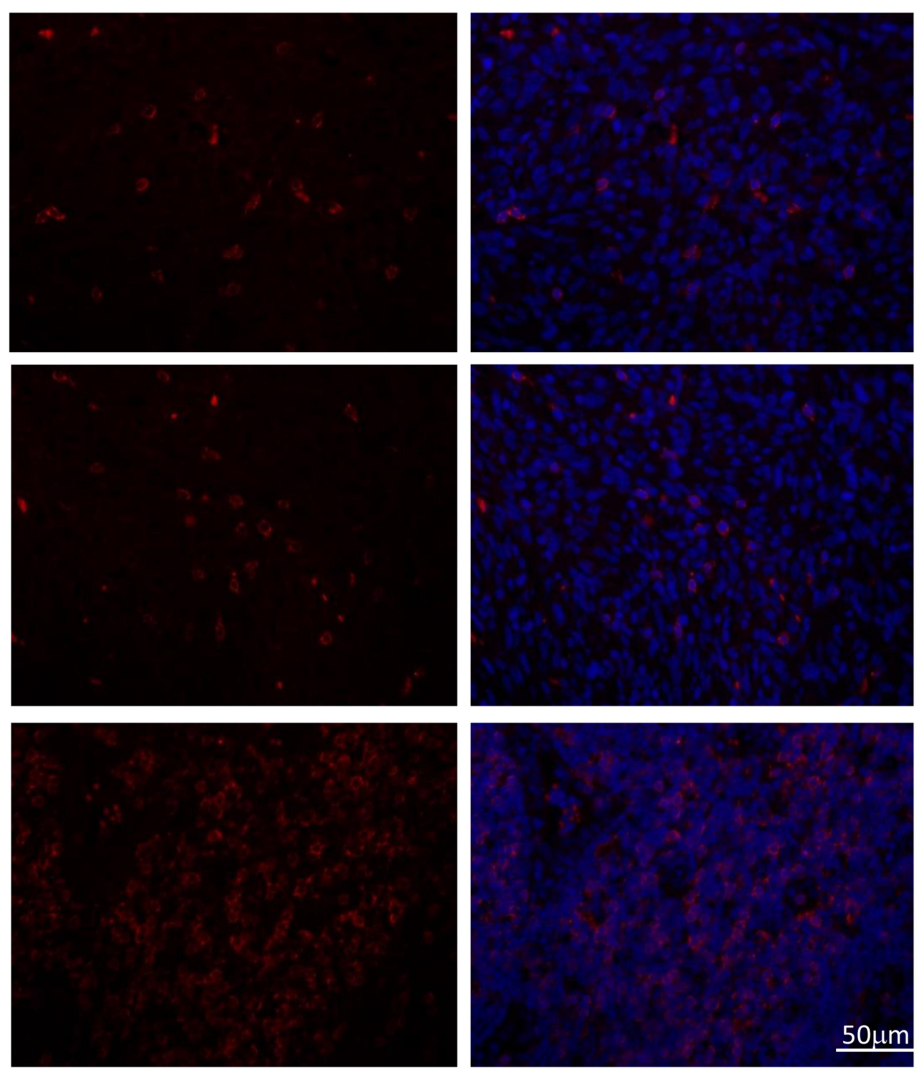

Figure 5 GQD-PEG-mediated photo-immune therapy. (A) Schematic overview of GQD-PEG-mediated photo-immune experimental workflow. (B) Tumor volume and (D) body weight curves of $\mathrm{C} 3 \mathrm{H}$ mice from the different groups; $(\mathbf{C})$ weight and $(\mathbf{E})$ images of SCC VII tumors at the end of the experiment; $(\mathbf{F})$ immunofluorescence staining of $C D 8 \mathrm{a}$ in the tumor tissues from mice after different treatments. $* * P<0.01 ; * * * * P<0.0001$ 
flow cytometry and the ELISA assay. The levels of the representative proinflammatory cytokines, including TNF- $\alpha$ and IFN- $\gamma$, were detected in serum by ELISA. The laser groups could stimulate the secretion of TNF- $\alpha$ and IFN- $\gamma$ compared with the control groups (Figure S6A). However, cytokine expression was significantly higher when treated with GQD-PEG under laser irradiation, which suggested that GQD-PEG could induce strong immune responses as a potential stimulator. ${ }^{11,39}$ Previous studies have demonstrated that $\mathrm{CD} 4^{+} \mathrm{T}$ helper cells could activate cytotoxic cells such as cytotoxic T cells and natural killer cells. Meanwhile, mature DCs present the antigen peptide complex to $\mathrm{CD}^{+}$cytotoxic $\mathrm{T}$ cells to form an MHC I-peptide complex, releasing cytotoxins (IFN- $\gamma$, perforin, granzymes, and granulysin) to kill cancer cells. $^{34,40}$ To examine whether GQD-PEG-mediated photoimmunotherapy could induce systemic immune responses, we evaluated the percentages of $\mathrm{CD}^{+} \mathrm{CD} 4^{+}$ $\mathrm{T}$ cells and $\mathrm{CD}^{+} \mathrm{CD}^{+} \mathrm{T}$ cells in tumor tissue at the end of the experiment. Flow cytometry results (Figure S6B) showed significantly increased percentages of $\mathrm{CD}^{+} \mathrm{CD}^{+} \mathrm{T}$ cells in the draining lymph tissues of the tumor. An immunofluorescence assay (Figure 5F) further confirmed the ability of GQDPEG with laser to promote $\mathrm{CD}^{+} \mathrm{T}$ cell infiltration into tumors. These results confirmed that the tumor areas of $\mathrm{C} 3 \mathrm{H}$ mice treated with GQD-PEG under irradiation prompted $\mathrm{CD}^{+}$cytotoxic $\mathrm{T}$ cell infiltration into the tumor tissue, enhancing the efficacy of its inhibition of tumor growth. Further, GQD-PEG did not cause any damage to the liver, lung, spleen, and kidney, which indicated its high biosafety to normal tissues (Figure S7). Above all, moderate GQD-PEGmediated photoimmunotherapy photo-killed the tumor and released tumor-associated antigens, efficiently activating $\mathrm{CD}^{+}$cytotoxic $\mathrm{T}$ cells with the help of cytotoxin-releasing TNF- $\alpha$ and IFN- $\gamma$, and resulting in systematic antitumor immunity. Although phototherapy could kill the tumor cells only upon irradiation, the more actively recruited immune cells may compensate for them during the continuing treatment $_{\text {cycle. }}{ }^{41-43}$

\section{Conclusion}

In this work, GQD-PEG was synthesized and exhibited excellent water stability, a strong capacity for photodynamic efficiency, and immunostimulatory activity. After irradiation, the high ROS from photodynamic toxicity killed tumor cells and triggered immune responses by releasing endogenous tumor antigens. Subsequently, inflammatory cytokine secretion (TNF- $\alpha$ and IFN- $\gamma$ ) induced the recruitment of cytotoxic $\mathrm{T}$ lymphocytes to tumors to strengthen therapeutic efficacy.
Moreover, fluorescence imaging demonstrated the tumortargeting accumulation of GQD-PEG in vitro and in vivo. This study illustrated that GQD-PEG is a promising candidate for the combination of photodynamic and immunotherapy.

\section{Acknowledgment}

This article was supported by the National Natural Science Foundation of China (No. 81873711).

\section{Disclosure}

The authors declare no competing financial interest and report no conflicts of interest for this work.

\section{References}

1. Siegel RL, Miller KD, Jemal A. Cancer statistics, 2020. CA Cancer J Clin. 2020;70(1):7-30. doi:10.3322/caac.21590

2. Zhang B, Du W, Gan K, Fang Q, Zhang X. Significance of the neutrophil-to-lymphocyte ratio in young patients with oral squamous cell carcinoma. Cancer Manag Res. 2019;11:7597-7603. doi:10.2147/CMAR.S211847

3. Nam J, Son S, Park KS, Zou WP, Shea LD, Moon JJ. Cancer nanomedicine for combination cancer immunotherapy. Nat Rev Mater. 2019;4(6):398-414. doi:10.1038/s41578-019-0108-1

4. Pauken KE, Dougan M, Rose NR, Lichtman AH, Sharpe AH. Adverse events following cancer immunotherapy: obstacles and opportunities. Trends Immunol. 2019;40(6):511-523. doi:10.1016/j. it.2019.04.002

5. Duan XP, Chan C, Lin WB. Nanoparticle-mediated immunogenic cell death enables and potentiates cancer immunotherapy. Angew ChemInt Edit. 2019;58(3):670-680. doi:10.1002/anie.201804882

6. Garg AD, Nowis D, Golab J, Agostinis P. Photodynamic therapy: illuminating the road from cell death towards anti-tumour immunity. Apoptosis. 2010;15(9):1050-1071. doi:10.1007/s10495-010-0479-7

7. Maeding N, Verwanger $T$, Krammer B. Boosting tumor-specific immunity using PDT. Cancers. 2016;8(10):91. doi:10.3390/ cancers 8100091

8. Wang D, Wang T, Liu J, et al. Acid-activatable versatile micelleplexes for PD-L1 blockade-enhanced cancer photodynamic immunotherapy. Nano Lett. 2016;16(9):5503-5513. doi:10.1021/acs.nanolett.6b01994

9. Yu X, Gao D, Gao L, et al. Inhibiting metastasis and preventing tumor relapse by triggering host immunity with tumor-targeted photodynamic therapy using photosensitizer-loaded functional nanographenes. ACS Nano. 2017;11(10):10147-10158. doi:10.1021/ acsnano. $7 \mathrm{~b} 04736$

10. Riley RS, June CH, Langer R, Mitchell MJ. Delivery technologies for cancer immunotherapy. Nat Rev Drug Discov. 2019;18(3):175-196. doi:10.1038/s41573-018-0006-z

11. Wu C, Guan X, Xu J, et al. Highly efficient cascading synergy of cancer photo-immunotherapy enabled by engineered graphene quantum dots/photosensitizer/CpG oligonucleotides hybrid nanotheranostics. Biomaterials. 2019;205:106-119. doi:10.1016/j. biomaterials.2019.03.020

12. Zhang X, Wei C, Li Y, Yu D. Shining luminescent graphene quantum dots: synthesis, physicochemical properties, and biomedical applications. Trends Anal Chem. 2019;116:109-121. doi:10.1016/j. trac.2019.03.011

13. Yu Y, Mei L, Shi Y, et al. Ag-conjugated graphene quantum dots with blue light-enhanced singlet oxygen generation for ternary-mode highly-efficient antimicrobial therapy. J Mater Chem B. 2020;8 (7):1371-1382. doi:10.1039/c9tb02300c 
14. Ge J, Lan M, Zhou B, et al. A graphene quantum dot photodynamic therapy agent with high singlet oxygen generation. Nat Commun. 2014;5:4596. doi:10.1038/ncomms5596

15. $\mathrm{Xu} \mathrm{A}, \mathrm{He} \mathrm{P}, \mathrm{Ye} \mathrm{C}$, et al. Polarizing graphene quantum dots toward long-acting intracellular reactive oxygen species evaluation and tumor detection. ACS Appl Mater Interfaces. 2020;12 (9):10781-10790. doi:10.1021/acsami.9b20434

16. Lesani P, Singh G, Viray CM, et al. Two-photon dual-emissive carbon dot-based probe: deep-tissue imaging and ultrasensitive sensing of intracellular ferric ions. ACS Appl Mater Interfaces. 2020;12 (16):18395-18406. doi:10.1021/acsami.0c05217

17. Wei Z, Yin X, Cai Y, et al. Antitumor effect of a Pt-loaded nanocomposite based on graphene quantum dots combats hypoxia-induced chemoresistance of oral squamous cell carcinoma. Int J Nanomed. 2018;13:1505-1524. doi:10.2147/IJN.S156984

18. Novak TG, Kim J, Song SH, et al. Fast P3HT exciton dissociation and absorption enhancement of organic solar cells by PEG-functionalized graphene quantum dots. Small. 2016;12 (8):994-999. doi:10.1002/smll.201503108

19. Mihalache I, Radoi A, Munteanu C, Kusko M, Kusko C. Charge storage and memory effect in graphene quantum dots-PEG(600) hybrid nanocomposite. Org Electron. 2014;15(1):216-225. doi:10.1016/j.orgel.2013.10.031

20. Li Y, Wu Z, Du D, Dong H, Shi D, Li Y. A graphene quantum dot (GQD) nanosystem with redox-triggered cleavable PEG shell facilitating selective activation of the photosensitiser for photodynamic therapy. RSC Adv. 2016;6(8):6516-6522. doi:10.1039/c5ra23622c

21. Cao Y, Dong H, Yang Z, et al. Aptamer-conjugated graphene quantum dots/porphyrin derivative theranostic agent for intracellular cancer-related microRNA detection and fluorescence-guided photothermal/photodynamic synergetic therapy. ACS Appl Mater Interfaces. 2017;9(1):159-166. doi:10.1021/acsami.6b13150

22. Li S, Zhou S, Li Y, et al. Exceptionally high payload of the IR780 iodide on folic acid-functionalized graphene quantum dots for targeted photothermal therapy. ACS Appl Mater Interfaces. 2017;9 (27):22332-22341. doi:10.1021/acsami.7b07267

23. Zhang X, Wei C, Li Y, et al. Dose-dependent cytotoxicity induced by pristine graphene oxide nanosheets for potential bone tissue regeneration. J Biomed Mater Res A. 2020;108(3):614-624. doi:10.1002/jbm.a.36841

24. Yaghini E, Turner H, Pilling A, Naasani I, MacRobert AJ. In vivo biodistribution and toxicology studies of cadmium-free indium-based quantum dot nanoparticles in a rat model. Nanomedicine. 2018;14 (8):2644-2655. doi:10.1016/j.nano.2018.07.009

25. Comparetti EJ, Pedrosa VA, Kaneno R. Carbon nanotube as a tool for fighting cancer. Bioconjug Chem. 2018;29(3):709-718. doi:10.1021/ acs.bioconjchem.7b00563

26. Goreham RV, Schroeder KL, Holmes A, Bradley SJ, Nann T. Demonstration of the lack of cytotoxicity of unmodified and folic acid modified graphene oxide quantum dots, and their application to fluorescence lifetime imaging of HaCaT cells. Mikrochim Acta. 2018;185(2):128. doi:10.1007/s00604-018-2679-8

27. Wang Y, Bi Q, Dong L, et al. Quinacrine enhances cisplatin-induced cytotoxicity in four cancer cell lines. Chemotherapy. 2010;56 (2):127-134. doi:10.1159/000313525

28. Li L, Cao B, Liang X, et al. Microenvironmental oxygen pressure orchestrates an anti- and pro-tumoral gammadelta $\mathrm{T}$ cell equilibrium via tumor-derived exosomes. Oncogene. 2019;38(15):2830-2843. doi:10.1038/s41388-018-0627-z
29. Korbelik M, Banath J, Zhang W, et al. Interaction of acid ceramidase inhibitor LCL521 with tumor response to photodynamic therapy and photodynamic therapy-generated vaccine. Int J Cancer. 2016;139 (6):1372-1378. doi:10.1002/ijc.30171

30. Shi S, Zhang L, Zhu M, et al. Reactive oxygen species-responsive nanoparticles based on PEGlated prodrug for targeted treatment of oral tongue squamous cell carcinoma by combining photodynamic therapy and chemotherapy. ACS Appl Mater Interfaces. 2018;10 (35):29260-29272. doi:10.1021/acsami.8b08269

31. Gondivkar SM, Gadbail AR, Choudhary MG, Vedpathak PR, Likhitkar MS. Photodynamic treatment outcomes of potentially-malignant lesions and malignancies of the head and neck region: a systematic review. J Investig Clin Dent. 2018;9(1). doi:10.1111/jicd.12270

32. Prazmo EJ, Kwasny M, Lapinski M, Mielczarek A. Photodynamic therapy as a promising method used in the treatment of oral diseases. Adv Clin Exp Med. 2016;25(4):799-807. doi:10.17219/acem/32488

33. Ren S, Cheng X, Chen M, et al. Hypotoxic and rapidly metabolic PEG-PCL-C3-ICG nanoparticles for fluorescence-guided photothermal/photodynamic therapy against OSCC. ACS Appl Mater Interfaces. 2017;9(37):31509-31518. doi:10.1021/acsami.7b09522

34. Rajendrakumar SK, Uthaman S, Cho CS, Park IK. Nanoparticlebased phototriggered cancer immunotherapy and its domino effect in the tumor microenvironment. Biomacromolecules. 2018;19 (6):1869-1887. doi:10.1021/acs.biomac.8b00460

35. Hou X, Tao Y, Pang Y, Li X, Jiang G, Liu Y. Nanoparticle-based photothermal and photodynamic immunotherapy for tumor treatment. Int J Cancer. 2018;143(12):3050-3060. doi:10.1002/ijc.31717

36. Saleem J, Wang L, Chen C. Carbon-based nanomaterials for cancer therapy via targeting tumor microenvironment. Adv Healthc Mater. 2018;7(20):e1800525. doi:10.1002/adhm.201800525

37. Tomic S, Janjetovic K, Mihajlovic D, et al. Graphene quantum dots suppress proinflammatory $\mathrm{T}$ cell responses via autophagy-dependent induction of tolerogenic dendritic cells. Biomaterials. 2017;146:13-28. doi:10.1016/j.biomaterials.2017.08.040

38. Tosic J, Stanojevic Z, Vidicevic S, et al. Graphene quantum dots inhibit $\mathrm{T}$ cell-mediated neuroinflammation in rats. Neuropharmacology. 2019;146:95-108. doi:10.1016/j.neuropharm.2018.11.030

39. Duan X, Chan C, Guo N, Han W, Weichselbaum RR, Lin W. Photodynamic therapy mediated by nontoxic core-shell nanoparticles synergizes with immune checkpoint blockade to elicit antitumor immunity and antimetastatic effect on breast cancer. $J$ Am Chem Soc. 2016;138(51):6686-16695. doi:10.1021/jacs.6b09538

40. Dong $\mathrm{H}, \mathrm{Su} \mathrm{H}$, Chen L, et al. Immunocompetence and mechanism of the DRibble-DCs vaccine for oral squamous cell carcinoma. Cancer Manag Res. 2018;10:493-501. doi:10.2147/CMAR.S155914

41. Song W, Kuang J, Li C-X, et al. Enhanced immunotherapy based on photodynamic therapy for both primary and lung metastasis tumor eradication. ACS Nano. 2018;12(2):1978-1989. doi:10.1021/ acsnano.7b09112

42. Li L, Yang S, Song L, et al. An endogenous vaccine based on fluorophores and multivalent immunoadjuvants regulates tumor micro-environment for synergistic photothermal and immunotherapy. Theranostics. 2018;8(3):860-873. doi:10.7150/ thno. 19826

43. Nam J, Son S, Ochyl LJ, Kuai R, Schwendeman A, Moon JJ. Chemophotothermal therapy combination elicits anti-tumor immunity against advanced metastatic cancer. Nat Commun. 2018;9(1):1074. doi:10.1038/s41467-018-03473-9 


\section{Publish your work in this journal}

The International Journal of Nanomedicine is an international, peerreviewed journal focusing on the application of nanotechnology in diagnostics, therapeutics, and drug delivery systems throughout the biomedical field. This journal is indexed on PubMed Central, MedLine, CAS, SciSearch ${ }^{\circledR}$, Current Contents ${ }^{\circledR} /$ Clinical Medicine, $^{2}$
Journal Citation Reports/Science Edition, EMBase, Scopus and the Elsevier Bibliographic databases. The manuscript management system is completely online and includes a very quick and fair peer-review system, which is all easy to use. Visit http://www.dovepress.com/ testimonials.php to read real quotes from published authors. 